\title{
Beyond Hearing Loss: Does Tinnitus Cause Cognitive Impairment?
}

\author{
Ho Yun Lee (iD \\ Department of Otorhinolaryngology-Head and Neck Surgery, Eulji University School of Medicine, Daejeon, Korea
}

It is commonly believed that tinnitus patients may have difficulties with attention span and memory. Many studies have reported that poor cognitive performance was associated with tinnitus. However, unlike hearing loss, which has been reported to be an independent risk factor for dementia, the link between tinnitus and cognitive impairment remains unclear [1].

Mild cognitive impairment (MCI) refers to an intermediate cognitive state in elderly individuals, somewhere between the state of those aging normally and those with dementia. Patients with MCI are known to have a higher risk of developing dementia. In a cross-sectional study, Lee et al. [2] attempted to determine whether there was a relationship between tinnitus and MCI, and established that higher tinnitus handicap inventory (THI) scores, reflecting tinnitus severity, were associated with lower scores on a Korean version of the Montreal Cognitive Assessment. Similarly, mean hearing levels and tinnitus severity were significant predictors of MCI. The authors [2] concluded that the cognitive function of aged tinnitus patients should be assessed as part of the initial work-up for tinnitus.

However, the study of Lee et al. [2] and its conclusions had some limitations. First, the hearing threshold was worse in the MCI group, and the mean hearing threshold showed a stronger relationship with $\mathrm{MCI}$ - as manifested by a higher odds ratiothan the THI score. Even worse, multicollinearity tests of mean hearing levels and tinnitus severity should have been performed, but were not. In addition, the absence of more objective tools weakens the conclusions that can be drawn from their study. Lastly, they did not compare changes in cognitive function before and after tinnitus treatment.

In a similar Chinese study, baseline characteristics (e.g., age, sex, educational level, and hearing threshold) did not show significant relationships with tinnitus severity [3]. In line with the findings of Lee et al., [2] that study also found that patients with severe tinnitus ( $\mathrm{THI} \geq 38$ ) exhibited distinct cognitive deficits, as evaluated using the Cognitive Abilities Screening Instrument. Moreover, tinnitus severity showed a strong correlation with the extent of cognitive impairment. They provided objective data with P300 event-related potentials, which reflect cognitive processing. Although the amplitudes of N2 and N3 were not significantly different, the latency values, which are associated with stimulus evaluation, revealed a clear difference according to the severity of tinnitus.

In contrast, an electroencephalographic study reported that cognitive changes in tinnitus patients were associated with changes in hippocampal activity, as well as activity in the anterior cingulate and insula [4]. The hippocampal area plays an important role in the persistence of tinnitus by updating auditory memory, and it is also related to cognitive dysfunction in patients with tinnitus. Interestingly, hearing loss was not correlated with any of the tested variables in their study. They assumed that this was because they did not test supra-clinical frequencies above $8 \mathrm{kHz}$.

Tinnitus is inextricable from auditory deafferentation. Therefore, a confounding effect of hearing loss on cognitive function in tinnitus patients is inevitable. A prospective, longitudinal, randomized controlled trial could be the most effective solution for this issue. It is true that some generators of tinnitus in the brain may share common resources with attention and memory generators in the brain, such as the prefrontal cortex, limbic system, and hippocampus $[5,6]$. However, it is too early to draw a firm conclusion that tinnitus can cause cognitive dysfunction.

\section{CONFLICT OF INTEREST}

No potential conflict of interest relevant to this article was reported.

\section{ORCID}

HoYun Lee https://orcid.org/0000-0002-9590-3477

Copyright (C) 2020 by Korean Society of Otorhinolaryngology-Head and Neck Surgery.

This is an open-access article distributed under the terms of the Creative Commons Attribution Non-Commercial License (https://creativecommons.org/licenses/by-nc/4.0)

which permits unrestricted non-commercial use, distribution, and reproduction in any medium, provided the original work is properly cited. 


\section{REFERENCES}

1. Lin FR, Metter EJ, O’Brien RJ, Resnick SM, Zonderman AB, Ferrucci L. Hearing loss and incident dementia.Arch Neurol. 2011 Feb;68(2): 214-20.

2. Lee SY, Lee JY, Han SY, Seo Y, Shim YJ, Kim YH. Neurocognition of aged patients with chronic tinnitus: focus on mild cognitive impairment. Clin Exp Otorhinolaryngol. 2020 Feb;13(1):8-14.

3. Wang Y, Zhang JN, Hu W, Li JJ, Zhou JX, Zhang JP, et al. The characteristics of cognitive impairment in subjective chronic tinnitus. Brain Behav. 2018 Jan;8(3):e00918.

4. Vanneste S, Faber M, Langguth B, De Ridder D.The neural correlates of cognitive dysfunction in phantom sounds. Brain Res. 2016 Jul;
1642:170-9

5. Tavanai E, Mohammadkhani G.A different view on the link between tinnitus and cognition; is there a reciprocal link? Int J Neurosci. 2018 Dec;128(12):1188-98.

6. Lee HY, Choi MS, Chang DS, Cho CS. Combined bifrontal transcranial direct current stimulation and tailor-made notched music training in chronic tinnitus. J Audiol Otol. 2017 Apr;21(1):22-7.

Received November 30, 2019 Accepted January 22, 2020 